\title{
CaM Kinase II and Visual Input Modulate Memory Formation in the Neuronal Circuit Controlling Courtship Conditioning
}

\author{
Mei-ling A. Joiner and Leslie C. Griffith \\ Department of Biology and Volen Center for Complex Systems, Brandeis University, Waltham, \\ Massachusetts 02254-9110
}

\begin{abstract}
In Drosophila, calcium/calmodulin-dependent protein kinase II (CaM kinase) has been shown to be important in the expression of both learning and memory for the associative behavior courtship conditioning. In this study we examine the role of visual input in producing this behavior and the effects of modifying visual input on CaM kinase-dependent memory formation. Inhibition of CaM kinase blocked apparent learning regardless of visual input. Visual input selectively affected the memory phase of courtship conditioning: normal visual input masked the memory effects of inhibition of CaM kinase resulting in generation of memory without apparent learning, whereas
\end{abstract}

disruption of visual input revealed the CaM kinase-dependence of memory. Visual input was found to be important only during the training period, which implies that vision and CaM kinase are interacting in the formation rather than the retrieval of memory. Our results suggest a model for courtship conditioning in which multiple sensory inputs are integrated at a CaMkinase-dependent neuronal switch to modulate courtship behavior.

Key words: CaM kinase II; Drosophila; courtship conditioning; memory; learning; visual mutants; transgenes
The generation of behavioral plasticity involves integration of inputs to produce changes in a specific output. The complete set of inputs and the mode and site of integration are often more difficult to determine than the change in output that defines the behavior. Using the powerful array of genetic methods available in Drosophila, it is possible to investigate the molecular mechanisms of behavior. In this study we define a role for visual input in courtship conditioning. Manipulation of visual input in a context of normal chemosensory input reveals CaM kinasedependent memory formation and suggests that multiplicity in sensory inputs for this behavior may be an important modulator of memory formation.

When presented with a female fly, male flies perform a mating ritual that includes orientation toward the female, wing extension and vibration to produce a courtship song, licking and eventually copulation (Spieth, 1974). These behaviors occur sequentially, and steps are not skipped. This courtship behavior was thought for many years to be "hard-wired": a behavioral program that could be turned on or off but with no plastic features. This assumption was challenged by the finding of Siegel and Hall (1979) that courtship could be modulated by experience. Specifically, it was shown that male flies could be conditioned to suppress courtship by exposure to a mated female. The effects of this conditioning could be seen for 2-3 hr. Presentation of either a mated female or a virgin female during this time period elicits a subnormal amount of courtship. Subsequent studies suggested that this was an associative behavior in which courtship by the male and an aversive pheromonal signal produced by the mated

Received July 8, 1997; revised Sept. 5, 1997; accepted Sept. 19, 1997.

This work was supported by National Institutes of Health Program Project Grant P01-322503 (L.C.G.). We thank Matt Bianchi for initiating the modeling of courtship conditioning, Larry Abbott for help in developing the model, and Adriana Villella for help with statistical analysis.

Correspondence should be addressed to Dr. Leslie Griffith, Department of Biology MS008, Brandeis University, 415 South Street, Waltham, MA 02254-9110.

Copyright (C) 1997 Society for Neuroscience $0270-6474 / 97 / 179384-08 \$ 05.00 / 0$ female were both required to get courtship suppression (Ackerman and Siegel, 1986; Tompkins et al., 1983). Production of this aversive signal is stimulated by courtship, and the strength of the signal increases with time (Gailey and Siegel, 1989; Tompkins and Hall, 1981; Tompkins et al., 1983).

Multiple sensory pathways are thought to contribute to male courtship behavior. Courtship is known to be modified by vision, olfaction, and tactile chemosensory inputs, but no single sensory input has been shown to be absolutely required for courtship (for review, see Tompkins, 1984). Flies that cannot see and cannot smell will court and copulate (Tompkins et al., 1980; Gailey et al., 1986). Flies that are in visual and olfactory, but not tactile, contact with a female will court (Gailey et al., 1986). Elimination of all three sensory modalities virtually abolishes mating (Gailey et al., 1986). These studies suggest that it is an integration of these multiple sensory cues that is important for generation of the behavior.

As a first step toward understanding the neuronal and biochemical substrates of plastic behavior in Drosophila, we have used genetic and environmental manipulation to dissect the roles of visual input and calcium/calmodulin-dependent protein kinase II (CaM kinase) in courtship conditioning. As we have reported previously (Griffith et al., 1993), inhibition of CaM kinase can affect both learning and memory. We have now found that the strength and quality of visual inputs can selectively affect the memory phase of this CaM kinase-dependent plastic behavior. Disruption of visual input enhances the effects of inhibition of CaM kinase on memory, but it does not alter the disruption of apparent learning produced by decreases in CaM kinase activity. Visual input masks the effects of CaM kinase inhibition on memory, resulting in animals that express memory without apparent learning. Our results suggest a model for courtship conditioning in which visual and chemosensory inputs are integrated at a CaM kinase-dependent neuronal switch that regulates male courtship behavior. 


\section{MATERIALS AND METHODS}

\section{Drosophila strains}

Fly cultures were kept at $25^{\circ} \mathrm{C}$ with a $12 \mathrm{hr}$ light/dark cycle on autoclaved cornmeal, yeast, sucrose, and agar food. The genetic background used for the behavior experiments was either from Canton-S or the line $w^{1118}$ (isoCJ1) a $w$, Canton-S isogenic stock (Yin et al., 1995). In the text $w$ refers to this allele. Unless stated otherwise, genotypes are as described in Lindsley and Zimm (1992). All females used in the study were of the genotype C(1)DX, ywf. The learning and memory response to these $y w f$ females was found to be equivalent to that obtained with Canton S wild-type females (M. A. Joiner, unpublished observations). The CaM kinase mutant CaMKII is described elsewhere (L. C. Griffith, M. A. Joiner, N. Kupiec, N. Marwaha, and M. Pla). Mutation at the CaMKII locus leads to a $40 \%$ decrease in CaM kinase activity and a $50 \%$ loss of CaM kinase protein by Western blot in heterozygotes. The ala inhibitor peptide is a synthetic peptide based on the sequences of the rat $\alpha$ CaM kinase autoregulatory domain (Griffith et al., 1993). Two methods of expression of ala are used in experiments presented in this paper: the hsp70-ala transgenic line (ala2), which is used without heat shock, and a UAS line that has the upstream activator sequences linked to the gene for the ala peptide. The GAL4/UAS system is described by Brand and Perrimon (1993). A new chromosome 1 neural GAL4 line (MJ85b) was generated by mating $F M 7 a$ females with a GAL4 insert on the balancer chromosome to $w ; \quad M K R S, \quad \Delta 2-3 / T M 2, \Delta 2-3$ males. FM7a,GAL4;MKRS, $\Delta 2-3$ or FM7a,GAL4;TM2, $\Delta 2-3$ virgin females were individually crossed to $w^{1118}$ (isoCJ1) males. MKRS is an inversioncontaining balancer chromosome. $w^{+}$, non- $F M 7 a$, nonmottled-eyed progeny, which represent new stable mobilizations of the GAL4 transposon, were singly crossed to $w^{1118}$ (isoCJ1) flies. MJ85b was chosen for this study because of its high level of expression in the central brain and low level of photoreceptor expression. F1 males that were tested in the learning assay resulted from crossing virgin females, homozygous for the GAL4 insert, to males homozygous for the UAS-ala insert. F1 males that were sectioned and stained resulted from crossing virgin females, homozygous for the GAL4 insertion, to males homozygous for a UAS-lacZ insert (Fischer et al., 1988), which expresses cytoplasmic $\beta$-galactosidase.

\section{Sectioning and staining}

GAL4;UAS-lacZ flies were put into a fly collar (Jager and Fischbach, 1987) so that their heads were aligned and facing the same way. Heads were frozen in Tissue Tek, and $12 \mu \mathrm{m}$ frontal cryostat sections were loaded onto Superfrost Plus slides. After the tissue was dried onto the slides, it was fixed $[1.5 \%(\mathrm{w} / \mathrm{v})$ glutaraldehyde, $2.0 \%(\mathrm{w} / \mathrm{v})$ formaldehyde, $38.0 \%(\mathrm{w} / \mathrm{v})$ sucrose, $1.0 \%(\mathrm{w} / \mathrm{v}) \mathrm{CaCl}_{2}, 1.0 \%(\mathrm{w} / \mathrm{v})$ gum arabic, and 0.05 $\mathrm{M}$ cacodylic acid, adjusted to $\mathrm{pH} 7.3$ ] for $10 \mathrm{~min}$ at room temperature, and then washed twice in $1 \times \mathrm{PBS}(0.02 \mathrm{M}$ sodium phosphate, $0.5 \mathrm{M} \mathrm{NaCl})$ for $10 \mathrm{~min}$ at room temperature. The slides were incubated overnight in the $\mathrm{X}$-gal staining solutions [8\% X-gal in DMSO diluted 1:30 in $\mathrm{Fe} / \mathrm{NaP}$ buffer, pH 7.2, $1.8 \mathrm{ml}$ of $0.2 \mathrm{M} \mathrm{Na}_{2} \mathrm{HPO}_{4}, 0.7 \mathrm{ml}$ of $0.2 \mathrm{M} \mathrm{NaH}_{2} \mathrm{PO}_{4}, 1.5$ $\mathrm{ml}$ of $5 \mathrm{M} \mathrm{NaCl}, 50 \mathrm{ml}$ of $1 \mathrm{M} \mathrm{MgCl}_{2}, 3.05 \mathrm{ml}$ of $50 \mathrm{~mm} \mathrm{~K}_{3}\left(\mathrm{Fe}(\mathrm{CN})_{6}\right), 3.05$ $\mathrm{ml}$ of $50 \mathrm{~mm} \mathrm{~K}_{4}\left(\mathrm{Fe}(\mathrm{CN})_{6}\right)$, distilled water to $50 \mathrm{ml}$ at $37^{\circ} \mathrm{C}$. After they were stained, the slides were rinsed twice for $10 \mathrm{~min}$ in distilled water and mounted (Crystal Mount, Biomeda Corporation, and Permount, Fisher Scientific, Houston, TX). Photographs were taken using Kodachrome T160 slide film using phase-contrast or Nomarski optics, and then scanned into Adobe Photoshop software on a Power Macintosh using a Polaroid Sprintscan.

\section{Behavior assays}

Courtship conditioning assay. Singly housed, 5-d-old test males were placed with 4-d-old $C(1) D X, y w f$ females, mated the previous day, in single-pair-mating chambers ( $8 \mathrm{~mm}$ diameter $\times 3 \mathrm{~mm}$ high $)$ for $1 \mathrm{hr}$. For each of the $10 \mathrm{~min}$ periods observed, a courtship index $(C I)$ was measured for each male tested. The fraction of time a male spends courting in a $10 \mathrm{~min}$ interval constitutes the $C I$. The first and last $10 \mathrm{~min}$ of this conditioning period were videotaped; $2-5 \mathrm{~min}$ after conditioning, the males were paired in a clean mating chamber with anesthetized virgin females collected that day, and the pairs were videotaped for the $10 \mathrm{~min}$ test period $\left(C I_{\mathrm{t}}\right)$. Learning was calculated by dividing the $C I$ for the final $10 \mathrm{~min}$ of the training hour $\left(C I_{\mathrm{f}}\right)$ by $C I$ for the initial $10 \mathrm{~min}$ of the training hour $\left(C I_{\mathrm{i}}\right)$. As a control, sham tests were performed in which the males were kept alone in the mating chamber for the first hour and then paired with anesthetized virgin females for the $10 \mathrm{~min}$ test period $\left(C I_{\text {sham }}\right)$. Because males used for the test period were not the same as those in the sham test, memory was measured as a comparison of $C I_{\mathrm{t}}$ with $C I_{\text {sham }}$. In some cases, to assess the response to a mated female tester, we then placed the test male with a mated female for an additional $10 \mathrm{~min}$ test period. The $C I$ calculated $\left(C I_{\mathrm{t} 2}\right)$ was compared with $C I_{\mathrm{f}}$. Experiments were performed at $25^{\circ} \mathrm{C}$ and $75 \%$ humidity in a Harris Environment Room. Two $52 \mathrm{~W}$ light bulbs, not pointed directly at the mating chamber, constituted white light conditions. Unless indicated otherwise, all behavior experiments were performed under red light (two lamps with $25 \mathrm{~W}$ red photographic light bulbs were placed $20 \mathrm{~cm}$ from the mating wheel). $n \geq 20$ for all genotypes.

Locomotor activity assay. Spontaneous locomotor activity was measured by counting the number of times a fly crosses a line drawn across an 8-mm-diameter, 3-mm-high circular chamber in a 4 min period.

\section{Statistics}

Each $C I$ was subjected to arcsine, arcsine squared, or arcsine square root transformation to effect approximation of normal distributions (Sokal and Rohlf, 1995; Villella and Hall, 1996) ANOVA with genotype or light condition as the main effect and subsequent Dunnett's test $(\alpha=0.05)$ were then performed on the transformed data using JMP software (version 3.1 for the Macintosh: SAS Institute, Inc.). Locomotor data were distributed normally and analyzed without transformation.

\section{RESULTS}

\section{Light, but not CaM kinase, modulates basal courtship behavior}

A number of investigators have noted that light and visual competence of the male have significant effects on mating success in flies as measured by sperm in the female reproductive tract or the number of females producing progeny (Geer and Green, 1962; Grossfield, 1966; Connolly et al., 1969; DeJianne et al., 1981; Tompkins et al., 1982; Markow, 1987; Chatterjee and Singh, 1988; Stocker and Gendre, 1989). To determine whether we could modulate baseline courtship behavior by changing visual input and whether modulation of basal courtship was affected by inhibition of CaM kinase, we assessed the amount of courtship performed by males with varying eye pigment levels and varying amounts of CaM kinase activity under normal white light and dim red light. Eye pigment levels were decreased by using flies carrying a mutation at the white $(w)$ locus, which eliminates all eye pigment and decreases visual acuity (Wehner et al., 1969). CaM kinase levels were modulated by expression of an autoinhibitory domain peptide inhibitor specific for CaM kinase (Griffith et al., $1993)$ under control of the hsp 70 promoter at $25^{\circ} \mathrm{C}($ ala $2 /+)$ or by heterozygosity for a mutation at the $C a M K I I$ locus. At $25^{\circ} \mathrm{C}$, the temperature at which all of our assays were performed, ala2/+ flies produce levels of inhibitor peptide sufficient to inhibit 15$25 \%$ of endogenous CaM kinase activity (Griffith and Greenspan, 1993). Mutation at the CaMKII locus leads to a $40 \%$ decrease in CaM kinase activity and a $50 \%$ loss of CaM kinase protein by Western blot in heterozygotes (L. C. Griffith, unpublished observations). Visual input was modulated by using dim red light. Flies are insensitive to light with wavelength $>650 \mathrm{~nm}$ (Frank and Zimmerman, 1969), so this condition is equivalent to assaying the flies in darkness but allows the experimenter to observe the behavior (Gailey et al., 1986; Stocker and Gendre, 1989; Hing and Carlson, 1996).

Figure 1 shows $C I$ for Canton-S (wild type), $w, w$; CaMKII/+, and $w$; ala2 $/+$ males assayed either in white or dim red light. $C I$ is the fractional amount of time spent in courtship activity during a 10 min observation period (Siegel and Hall, 1979; Griffith et al., 1993). Data for all experiments were compared using one-way ANOVA with either genotype or light condition as the main effect as indicated. Wild-type males with full eye pigmentation have a higher mean $C I$ than males with less eye pigment in white 


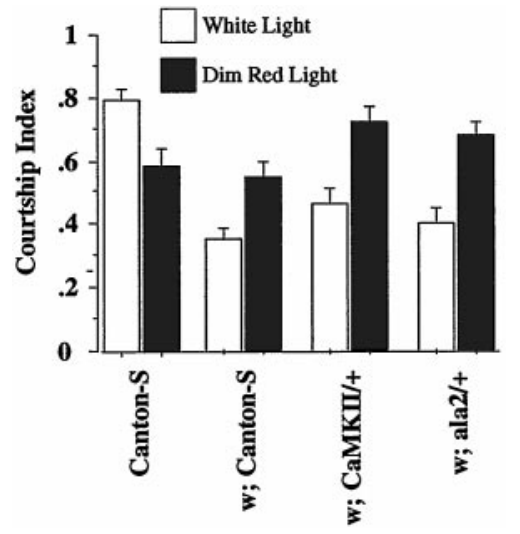

Figure 1. Light affects basal male courtship. The courtship index (CI), the fractional amount of the $10 \mathrm{~min}$ observation spent in courtship activity, for the initial $10 \mathrm{~min}$ of exposure of males of the indicated genotypes to mated females is shown. For each genotype, $C I$ was measured in white light (white bars) and dim red light (gray bars). Data are expressed as mean \pm SEM. $n \geq 20$ for all genotypes and conditions. For each genotype there is a statistically significant difference in courtship in the two light conditions.

light ( $p<0.05$ for all genotypes using Dunnett's test comparison to Canton-S). When the same comparison is made in dim red light, which decreases visual input for all genotypes, Canton-S males do not perform significantly better than flies with decreased eye pigment and/or inhibited CaM kinase $(p>0.05$ for all genotypes compared with Canton-S using Dunnett's test). This result is based on changes in the behavior of both the pigmented and hypopigmented flies. Males with no eye pigment ( $w$ or $w$; CaMKII/+) and males carrying a mini- $w+$ transgene that express subnormal amounts of eye pigment $(w ;$ ala $2 /+)$ are all inhibited by white light, showing significant increases in $C I$ under dim red light ( $p<0.001$ for $w, w ; C a M K I I /+$ and $w$; ala $2 /+$ ). Canton-S flies, in contrast, are inhibited by dim red light, showing a decrease in $C I(p<0.0001)$. Thus for pigmented flies, $C I$ goes down in darkness, but for hypopigmented animals, $C I$ increases. These results are consistent with results of mating competition studies (Geer and Green, 1962; Connolly et al., 1969) and also demonstrate that inhibition of CaM kinase or decreased CaM kinase levels do not change the effect of visual input on basal male courtship behavior. This indicates that even animals with decreased CaM kinase activity can respond normally to the modulatory effects of light and suggests that the effects of CaM kinase are not simply mediated by a change in the visual inputs that enhance courtship behavior. The apparently contradictory effects of light on pigmented versus unpigmented animals may be partly the result of changes in female behavior that allow increased success for visually impaired males in the dark; that is, the absence of visual input to courted females enhances their receptivity (Tompkins et al., 1982; Markow, 1987).

\section{Inhibition of CaM kinase blocks apparent learning, but not memory, in visually competent flies}

To study the plastic aspects of male courtship, we used a courtship conditioning paradigm. Apparent learning was measured by the decrement of courtship behavior displayed by the male during a 1 hr exposure to a mated female. The decrement is expressed as a ratio of the $C I$ for the final $10 \mathrm{~min}$ of the training period $\left(C I_{\mathrm{f}}\right)$ to the $C I$ for the initial 10 min of training $\left(C I_{\mathrm{i}}\right)$. For all lines tested, $C I_{\text {in }}$ was between 0.350 and 0.888 . The mean for control lines was 0.615 , and the mean for experimental lines was 0.704. Because

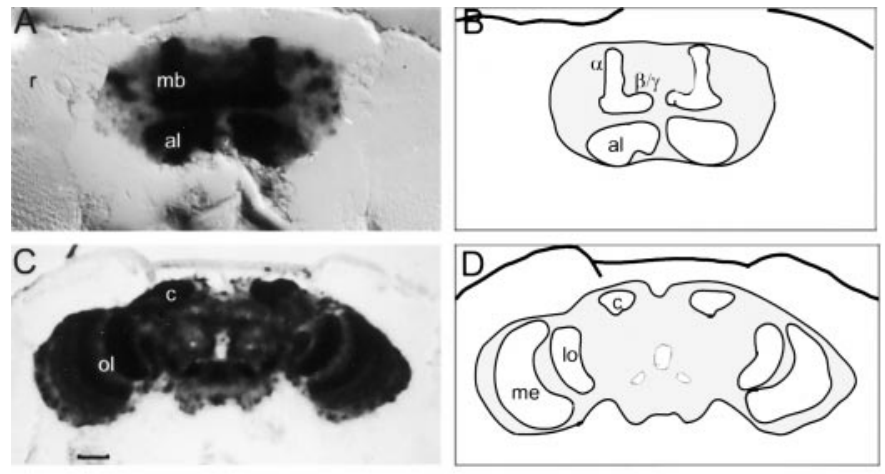

Figure 2. Expression pattern of GAL4 in line MJ85b. Expression of GAL4 was visualized by mating homozygous $M J 85 b$ virgin female flies to a line homozygous for $U A S$-lacZ. Frontal sections $(12 \mu \mathrm{m})$ of adult heads were stained with $\mathrm{X}$-gal to mark cells that contain $\beta$-galactosidase activity. The $M J 85 b$ line expresses in all neurons of the adult head, with the exception of photoreceptors of the retina $(r)$. Expression is most intense in the optic lobe $(\mathrm{ol})$, antennal lobe $(\mathrm{al})$, mushroom body lobe $(\mathrm{mb})$, and calyx (c) neuropils. $A$, Anterior brain; $B$, schematic diagram of anterior brain. Antennal lobe $(a l)$ and lobes of the mushroom bodies $(\alpha, \beta$, and $\gamma)$ are indicated. Shaded area indicates X-gal staining. $C$, Posterior brain. Scale bar, $50 \mu \mathrm{m}$. $D$, Schematic diagram of posterior brain. Medulla $(m e)$, calyx $(c)$, and lobula $(l o)$ are indicated. Shaded area indicates X-gal staining.

baseline courtship values will be understandably variable depending on genotype (Fig. 1 and see legends to Figs. 3 and 4), a ratio is used as a baseline-independent indicator of the magnitude of learning. For wild-type males this ratio is $\sim 0.5$ (see Fig. 3). Memory of conditioning was assessed by measuring the amount of courtship toward a virgin female after training with the mated female. A $C I\left(C I_{\mathrm{t}}\right)$ was calculated for a 10 min test with a virgin female immediately after conditioning. This value was compared with that obtained with a male that was "sham"-conditioned, i.e., spent $1 \mathrm{hr}$ in a chamber with no mated female $\left(C I_{\text {sham }}\right)$. For wild-type males, $C I_{\mathrm{t}}$ was significantly less than $C I_{\text {sham }}$.

Previous studies on the role of CaM kinase in courtship conditioning were performed using a transgene coding for a $\mathrm{CaM}$ kinase inhibitor peptide under control of the hsp70 promoter at $25^{\circ} \mathrm{C}$ under normal lighting conditions (Griffith et al., 1993, 1994). Both experimental and control lines had less than normal eye pigmentation and were therefore visually abnormal. To further study the role of CaM kinase in courtship conditioning, we sought additional methods for disrupting or decreasing enzyme activity in the adult nervous system in a context of normal visual function. We used three methods to achieve global decreases in $\mathrm{CaM}$ kinase activity: mutation of the endogenous CaMKII locus, expression of ala peptide at $25^{\circ} \mathrm{C}$ under hsp 70 promoter control, and global brain expression of the ala peptide under control of a GAL4 enhancer trap element. The expression pattern of the GAL4 line that we generated and used, MJ85b, is shown in Figure 2. GAL4 is expressed throughout the central brain and optic lobes but not in the photoreceptor cells. The intensity of $\beta$ galactosidase staining is high throughout the brain, being apparently highest in mushroom bodies, optic lobes, and antennal lobes. Because all of our transgenic animals were made using mini- $w^{+}$ as a P-element transformation marker, we replaced the mutant $w$ chromosome 1 in these lines by outcrossing to Canton-S, which is $w^{+}$, to generate visually competent males with full eye pigmentation for behavioral assays.

Figure $3 A$ shows data for apparent learning of visually compe- 
$\mathbf{A}$
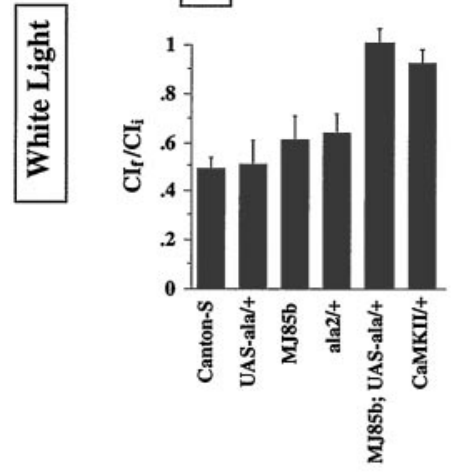

C

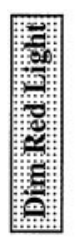

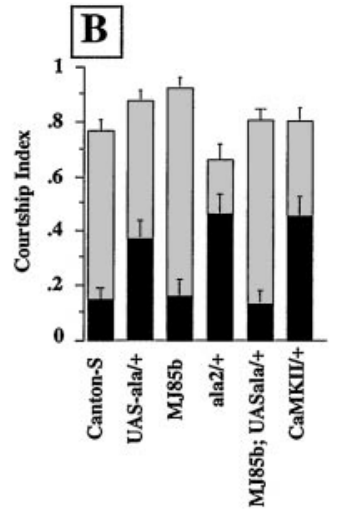



Figure 3. Learning and memory for flies with intact vision. Males of the indicated genotype (all of which have normal eye pigmentation) were trained by exposure to a mated female for $1 \mathrm{hr}$ and then immediately placed with an anesthetized virgin female. For sham training, flies of the same genotype were manipulated identically, except that no female was present in the chamber during training. All manipulations were performed at $25^{\circ} \mathrm{C}, 75 \%$ relative humidity. $A$, Conditioning in white light. Apparent learning was measured by calculating a courtship index $(C I)$ for the first $\left(C I_{\mathrm{i}}\right)$ and last $\left(C I_{\mathrm{f}}\right) 10 \mathrm{~min}$ of the conditioning period. Data are expressed as the ratio of means, $C I_{\mathrm{f}} / C I_{\mathrm{i}} \pm \mathrm{SEM}$. For wild-type flies (Canton-S) this value is $\sim 0.5$. Values $>0.5$ indicate defects in apparent learning, with a failure to decrease courtship during the training period. $C I_{\mathrm{i}}$ for each genotype was Canton-S, $0.82 \pm 0.23 ; U A S$-ala $/+, 0.71 \pm 0.23$; MJ85b, $0.79 \pm 0.26 ;$ ala $/+, 0.71 \pm 0.23 ;$ MJ85b; UAS-ala/+, $0.86 \pm 0.18$; CaMKII/+, $0.89 \pm 0.19 . B$, Memory in white light. Memory was tested by measuring a $C I$ for the initial $10 \mathrm{~min}$ of exposure to an anesthetized virgin female after training with a mated female (dark bars) or sham training (light bars). Data are expressed as mean \pm SEM. $C$, Conditioning in dim red light. Learning was measured as in $A$, with the exception that all procedures were performed in dim red light. Apparent learning in dim red light was not significantly different from that measured in white light. $C I_{\mathrm{i}}$ for each genotype was Canton-S, $0.56 \pm 0.26 ; U A S-a l a /+, 0.69 \pm 0.21$; MJ85b, $0.74 \pm 0.23 ;$ ala $2 /+, 0.80 \pm 0.17 ;$ MJ85b; UAS-ala/,$+ 0.71 \pm 0.25 ;$ CaMKII/+, $0.82 \pm 0.21$. D, Memory in dim red light. Memory was assessed as in $B$, with the exception that training and testing were performed in dim red light. $n \geq 20$ for all genotypes and conditions.

tent flies with full eye pigmentation in normal white light. $U A S$ ala $/+$ and $M J 85 b$ controls were not significantly different from Canton-S ( $p>0.05$ using Dunnett's test), indicating that there is no insertional effect of these P-elements in this assay. Males that have reduced $\mathrm{CaM}$ kinase activity $(M J 85 b ; U A S$-ala/+ and CaMKII/+) show poor apparent learning compared with Canton-S (using Dunnett's test; $p<0.05$ for all genotypes).

Memory of conditioning in white light for visually competent flies is shown in Figure $3 B$. Memory was measured as a difference between courtship of a virgin female after exposure to a mated

female (dark bars) or after sham conditioning in a chamber with no female (light bars). Sham conditioning produces equivalent results in all genotypes in white light $(p>0.05$ using Dunnett's test for all genotypes compared with Canton-S). For Canton-S wild type, there is a significant difference $(p<0.0001)$ between conditioned and sham-treated males. UAS-ala/+ and MJ85b controls also show normal memory ( $p<0.0001$ for both). Surprisingly, males with reduced CaM kinase activity also have fairly normal memory ( $p<0.02$ for ala2/+, CaMKII/+ and MJ85b; $U A S$-ala/+) even in the apparent absence of learning (Fig. $3 A$ ). The absolute difference between sham and conditioned $C I$, however, suggests that $C a M K I I /+$ and ala $2 /+$ have a partial memory phenotype. Although there is a statistically significant difference between sham and trained flies, the magnitude of this difference is less than the difference seen with control animals. Thus for flies with normal vision, inhibition of CaM kinase blocks apparent learning but leaves memory formation intact. The memory formed under these conditions is less robust.

\section{Lack of visual input unmasks the memory effects of inhibition of CaM kinase}

To assess the importance of vision we assayed flies in dim red light. Conditioning in dim red light (Fig. $3 C$ ) was not significantly different from conditioning in white light (Fig. $3 A$ ) for any of the genotypes ( $p \geq 0.1$ for all genotypes except $U A S$-ala $/+$ for which $p=0.002$ ). Memory of conditioning in dim red light, which blocks visual input for visually competent flies, is shown in Figure $3 D$. Sham conditioning was equivalent in all genotypes $(p<0.05$ using Dunnett's test comparing all genotypes to Canton-S). For Canton-S, UAS-ala/+, and MJ85b controls, memory was still normal ( $p<0.0001$ for both), although perhaps not as strong as that seen in white light. For animals with inhibited CaM kinase, however, memory was much more affected when assayed in dim red light, with the effect being largest for the MJ85b; UAS-ala/+ animals (ala2/+, $p<0.01 ;$ MJ85b; UAS-ala $/+, p>0.1)$. Memory remained intact in $C a M K I I /+$ animals $(p<0.0001)$. Thus for animals that are visually competent, turning off the lights increases the sensitivity of memory to $\mathrm{CaM}$ kinase inhibition. Interestingly, visual input had no effect on the learning phase of the assay. CaM kinase inhibition blocks apparent learning in both white and dim red light.

\section{Visual mutations unmask CaM kinase-dependent memory}

The above results suggested that in flies with subnormal vision as a result of mutations that affect the visual pathway, inhibition of CaM kinase should produce significant effects on memory in addition to effects on apparent learning. Furthermore, these effects should be light-independent. To genetically disrupt vision we used mutations in two genes: white $(w)$ and optomotor blind $\left(o m b^{\mathrm{H} 31}\right)$. Figure $4 A, C$ shows data for conditioning of visually impaired flies in normal and dim red light, respectively. Lines that are visually defective but have normal CaM kinase activity ( $w$ Canton-S and $o m b^{\mathrm{H} 31}$ ) conditioned normally, with $C I_{\mathrm{f}} / C I_{\mathrm{i}}$ being close to 0.5. In this study, and in previous ones (Siegel and Hall, 1979), light did not significantly affect conditioning for any line ( $p>0.01$ for all lines).

Figure $4 B, D$ shows data for memory of visually impaired flies in normal and dim red light, respectively. Memory was intact for flies with impaired vision but normal levels of CaM kinase ( $w$-Canton-S and $o m b^{\mathrm{H} 31} ; p<0.0001$ ). However, flies that have both reduced CaM kinase activity and impaired vision showed 
$\mathbf{A}$
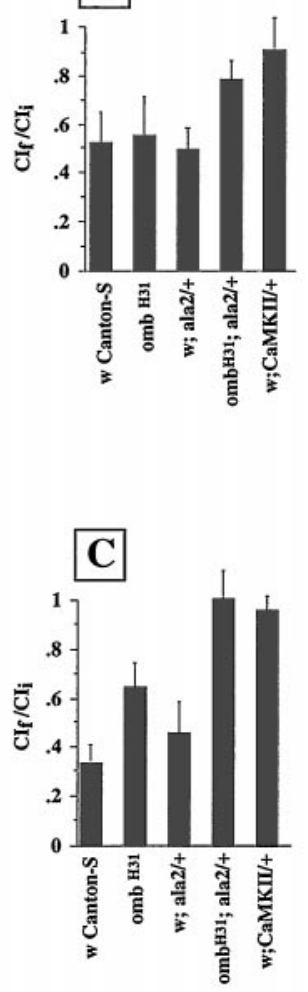

B

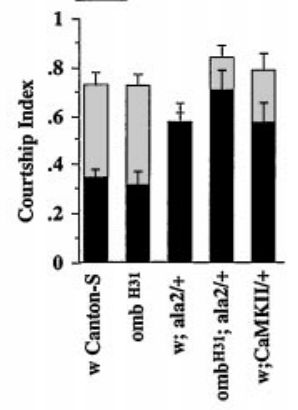

D

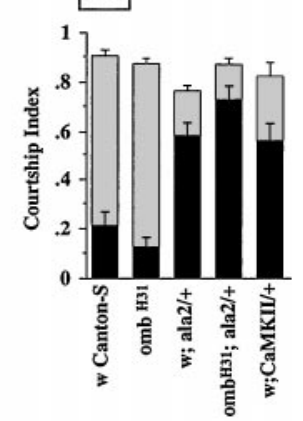

Figure 4. Learning and memory for visually impaired flies. Conditions for training and testing are described in the legend for Figure 3. A, Conditioning in white light. Apparent learning was measured by calculating a courtship index $(C I)$ for the first $\left(C I_{\mathrm{i}}\right)$ and last $\left(C I_{\mathrm{f}}\right) 10 \mathrm{~min}$ of the conditioning period. Data are expressed as the ratio of means, $C I_{\mathrm{f}} / C I_{\mathrm{i}} \pm$ SEM. $C I_{\mathrm{i}}$ for each genotype was $w$; Canton-S, $0.35 \pm 0.15 ; o m b^{\mathrm{H} 31}, 0.47 \pm$ $0.21 ; w ;$ ala $2 /+, 0.40 \pm 0.23 ; \mathrm{omb}^{\mathrm{H} 31} ;$ ala $2 /+, 0.84 \pm 0.11 ; w ; C a M K I I /+$, $0.46 \pm 0.24$. $B$, Memory in white light. Memory was tested by measuring a $C I$ for the initial $10 \mathrm{~min}$ of exposure to an anesthetized virgin female after training with a mated female (dark bars) or sham training (light bars). Data are expressed as mean $\pm \mathrm{SEM}$. $C$, Conditioning in dim red light. Learning was measured as in $A$, with the exception that all procedures were performed in dim red light. Apparent learning in dim red light was not significantly different from that measured in dim white light. $C I_{\mathrm{i}}$ for each genotype was $w$; Canton-S, $0.52 \pm 0.24 ;$ omb $^{\mathrm{H} 31}, 0.42 \pm 0.19 ; w$; ala $2 /+, 0.67 \pm 0.19 ;$ omb $^{\mathrm{H} 31} ;$ ala $2 /+, 0.64 \pm 0.15 ; w ; C a M K I I /+, 0.71 \pm$ 0.22 . $D$, Memory in dim red light. Memory was assessed as in $B$, with the exception that training and testing were performed in $\operatorname{dim}$ red light. $n \geq$ 20 for all genotypes and conditions.

partial memory decrements in both normal $(w$; ala $2 /+, p>0.8$; $o m b^{\mathrm{H} 31} ;$ ala $\left.2 /+, p>0.4 ; w ; C a M K I I /+, p>0.02\right)$ and dim red light $\left(w ;\right.$ ala $2 /+, p>0.001 ;$ omb $^{\mathrm{H} 31} ;$ ala $2 /+, p>0.07 ; w$; $C a M K I I /+, p>0.004)$. These results are consistent with the idea that disruption of visual input can unmask $\mathrm{CaM}$ kinasedependent memory formation.

The counterintuitive effects of manipulation of CaM kinase on the learning and memory phases of the assay (memory without apparent learning) are not attributable to differential effects on responses to mated as opposed to virgin females. If mated females are used in the memory test, results are similar to those obtained with virgin testers (Kane et al., 1997). Even in the absence of apparent learning, $w$; ala $2 /+$ flies expressed significant memory during the test phase if assayed with virgins (Fig. $4 B$ ) or if subsequently tested with mated females $(C I=0.20 \pm 0.08 ; n=$ $13 ; p<0.002$ compared with sham test of same genotype).
Table 1. Spontneous locomotor activity

\begin{tabular}{lc} 
Genotype & Line crossing \\
\hline Canton S & $128 \pm 10$ \\
$w ;$ Canton-S & $105 \pm 7$ \\
omb $b^{\text {H31 }}$ & $117 \pm 6$ \\
UAS-ala/+ & $151 \pm 6$ \\
MJ85b & $99 \pm 9$ \\
ala2/+ & $130 \pm 10$ \\
$w ;$ ala2/+ & $160 \pm 8$ \\
omb & $156 \pm 4$ \\
CaMKII/+ $/$ ala2/+ & $124 \pm 10$ \\
$w ;$ CaMKII/+ & $123 \pm 14$ \\
MJ85b; UAS-ala/+ & $130 \pm 5$
\end{tabular}

$\overline{\text { Locomotor activity was measured by the number of times flies cross a line drawn }}$ across the middle of an $8 \mathrm{~mm}$ chamber during a 4 min observation period. Assay was performed in dim red light; $n=10$ for all genotypes. Data are presented as mean \pm SEM.

Locomotor defects are also not the basis of these phenotypes. Assessment of spontaneous locomotor activity in these lines reveals no significant differences $(p>0.05$ using Dunnett's test compared with Canton-S wild type) (Table 1).

\section{Visual input is important for memory formation, not retrieval}

In each of the above experiments, conditioning and testing were performed in identical light conditions. To determine when visual input was important, during conditioning (for formation of memory) or during the virgin test (for retrieval of the memory), we performed an experiment in which MJ85b; UAS-ala/+ males were trained in one light condition and tested in the other. This genotype is visually intact and shows significant differences in memory, depending on the light conditions (Fig. 3). As shown in Figure 5, animals trained in white light and then tested in dim red light $(\mathrm{W} \rightarrow \mathrm{R})$ behaved identically to animals trained and tested in white light, and they had intact memory $(p<0.0001$ for comparison of $C I_{\mathrm{t}}$ to $\left.C I_{\text {sham }}\right)$. Animals trained in dim red light and then tested in white light $(\mathrm{R} \rightarrow \mathrm{W})$ behaved identically to animals trained and tested in dim red light, showing significant memory impairment ( $p>0.3$ for comparison of $C I_{\mathrm{t}}$ to $\left.C I_{\text {sham }}\right)$. This demonstrates that visual input is important only during the conditioning period for memory formation and is not relevant during expression of the memory.

\section{DISCUSSION}

The major conclusions of this study allow us to begin to formulate a model of how sensory inputs are integrated to achieve behavioral plasticity in male courtship. First, in visually intact animals, inhibition of CaM kinase predominantly affects the learning phase, resulting in a failure of the male to exhibit a decrement in courtship. This failure to "express" learning is not equivalent to an absence of actual learning as measured by the response to the subsequently presented virgin or mated female. This type of effect has also been reported for animals with inhibited protein kinase C (Kane et al., 1997). Second, visual input and CaM kinase activity interact to allow formation of memory. In the absence of normal visual input, inhibition of CaM kinase is much more effective in blocking memory formation. Third, the effects of visual input are specific to memory formation and not retrieval, because light conditions during training determine the outcome of the memory assay in cases where there are light-dependent 


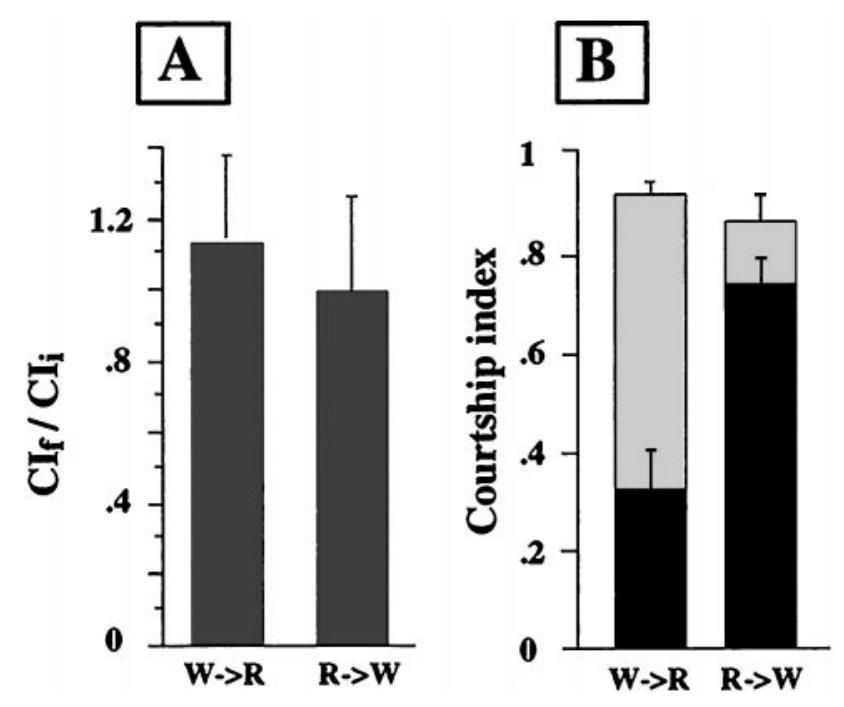

Figure 5. Visual input during conditioning determines memory phenotype in flies with inhibited CaM kinase. MJ85b; UAS-ala/+ males were trained by exposure to a mated female for $1 \mathrm{hr}$ in either white or dim red light and then immediately placed with an anesthetized virgin female in the opposite light condition. For sham training, flies and lights were manipulated identically, except that no female was present in the chamber during training. All manipulations were performed at $25^{\circ} \mathrm{C}, 75 \%$ relative humidity. $A$, Conditioning in white light for flies tested in dim red light $(W \rightarrow R)$ and conditioning in dim red light for flies tested in white light $(R \rightarrow W)$. Data are expressed as the ratio of means, $C I_{\mathrm{f}} / C I_{\mathrm{i}} \pm \mathrm{SEM}$. For wild-type flies (Canton-S) this value is $\sim 0.5$. Values $>0.5$ indicate defects in the apparent learning. $B$, Memory for flies conditioned in white light and then tested in dim red light $(W \rightarrow R)$ and for flies conditioned in dim red light and then tested in white light $(R \rightarrow W)$. Memory was tested by measuring a $C I$ for the initial $10 \mathrm{~min}$ of exposure to an anesthetized virgin female after training with a mated female (dark bars) or sham training (light bars). Data are expressed as mean \pm SEM. $n \geq 20$ for all genotypes and conditions.

memory phenotypes. Fourth, the effects of CaM kinase are at an integration step in the circuit, not in the visual input pathway itself, because inhibition of CaM kinase does not disrupt the nonplastic response to light. These conclusions have bearing on both the role of vision in courtship conditioning and the role of CaM kinase in plasticity.

\section{The role of vision in male sexual behavior}

Vision has been shown to increase the amount of courtship (Gailey et al., 1986) and the success rate of courtship (Markow, 1987). A modulatory role for vision has also been noted for courtship conditioning. Darkness or mutations in the visual transduction cascade decreased the robustness of the response, but males were still able to be conditioned (Siegel and Hall, 1979). All of these results are consistent with our findings that in normal and CaM kinase-deficient animals, light can modulate basic courtship behavior (Fig. 1).

In this study we use three independent methods of modulating visual input: dim red light, mutation at the $w$ locus, and mutation at the $o m b$ locus. Neither $w$ nor omb flies are completely blind. Both have visual impairments that decrease acuity (Wehner et al., 1969; Heisenberg et al., 1978). Our results suggest that light detection itself may not be the integrating factor, but rather processed visual information that triggers optomotor behaviors may be what is important. This is supported by studies showing that female movement increases courtship over that seen with immobile females (Tompkins et al., 1982), even with visually competent males. Male "tracking," a courtship-specific visual behavior, may be the critical determinant. Males with decreased acuity (e.g., $w$ or $o m b^{\mathrm{H} 31}$ ) cannot track accurately, even though light is being sensed (Cook, 1980), and consequently compete less well for females (Geer and Green, 1962) and give a lower CI (Fig. 1).

The White protein is a tryptophan/guanine transporter that is important for localization of eye pigment precursors that may also be important for neurotransmitter synthesis (Pepling and Mount, 1990). This raises the possibility that in $w$ flies, the defects we have seen are developmental. However, when tested in the dark or under conditions that decrease visual input (dim red lights), the courtship advantage of males with normal eye pigmentation over $w$ males is nullified (Fig. 1) (Isono, 1993). This suggests that there is no developmental effect on courtship by the $w$ mutation itself, but that it is acting primarily via its acute role in vision. All visual disruptions have the same effect on memory, unmasking the ability of CaM kinase inhibition to decrease memory. None of the visual manipulations, however, modulate the inhibition of apparent learning produced by decreased CaM kinase.

\section{The role of CaM kinase in courtship conditioning}

Disruption of CaM kinase activity was also accomplished in three independent ways: an hsp70-driven ala inhibitory peptide gene (ala2), heterozygosity for a mutation at the CaMKII locus, and GAL4-driven brain expression of the ala inhibitory peptide gene. The ala 2 transgene has been extensively characterized (Griffith et al., 1993, 1994; Wang et al., 1994). At $25^{\circ} \mathrm{C}$ it produces inhibitor peptide at levels capable of inhibiting only $15-25 \%$ of the endogenous CaM kinase activity (Griffith et al., 1993). This level of peptide is not high enough to appreciably inhibit either cAMPdependent protein kinase or protein kinase $\mathrm{C}$. In the case of $M J 85 b$; UAS-ala/+, the memory phenotype is similar to that produced by the ala 2 transgene, with no additional features. All three strategies for decreasing $\mathrm{CaM}$ kinase activity produce defects in expression of learning that are independent of light (Figs. 3 , 4). Interestingly, only peptide inhibition of the kinase has significant effects on memory. Decreasing the amount of kinase by mutating one copy of the gene $(\mathrm{CaMKII/+})$ does not affect memory, even in the absence of visual input.

Why does the CaMKII/+ mutant behave differently than the inhibitor flies? One possibility is that the ala peptide is inhibiting an additional kinase. One potential target is protein kinase C. This seems unlikely, however, because measured levels of peptide in ala 2 are not high enough to inhibit protein kinase $\mathrm{C}$ (Griffith et al., 1993). Another possible target would be other members of the CaM kinase family. To date there have been no indications that there are other CaM kinase II homologs in Drosophila. By Southern blot only one gene has been seen (Cho et al., 1991), and low-stringency screening of head cDNA libraries did not identify any other CaM kinase II homologs (L. C. Griffith, unpublished observations). As yet, CaM kinase I and IV have not been cloned from Drosophila. Another family member, the product of the caki gene, has been reported to be involved in locomotor behavior (Martin and Ollo, 1996). It seems unlikely that the peptide is inhibiting this kinase, however, because locomotor behavior of the lines used in this study appears normal (Table 1) (Griffith et al., 1993).

A second interesting possibility for the difference in effects seen between inhibition of the kinase and mutation at the CaMKII locus is that kinase protein level is also important in some way that is not correlated with catalytic activity. CaMKII/+ animals have both reduced kinase activity and reduced protein levels as 


\section{Serial Model}
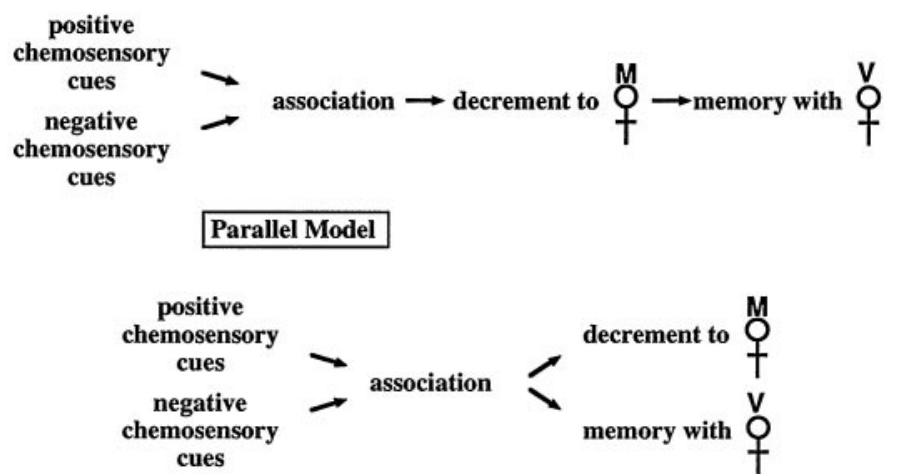

\section{Network Model}

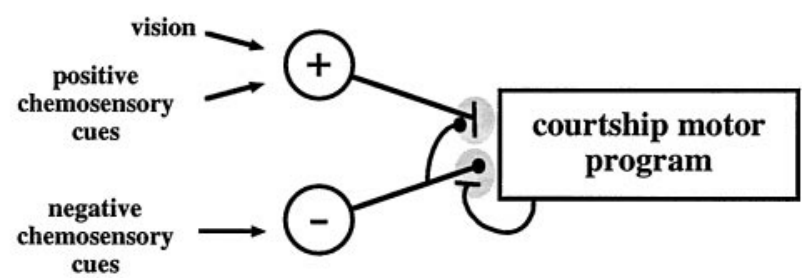

Figure 6. Models of courtship conditioning. Previous and current models of courtship conditioning are depicted. In the network model, a positive pathway responsive to both visual input and stimulatory pheromone and a negative pathway responsive to aversive pheromone are connected to the output center controlling male courtship. - indicates an excitatory connection; - indicates an inhibitory connection. Gray shading indicates connections at which $\mathrm{CaM}$ kinase-dependent heterosynaptic plasticity is expressed in the positive and negative pathways. Rules for synaptic strength modulation are as follows: (1) concurrent activity in the negative pathway and the courtship motor center leads to strengthening of the connection between the negative pathway and the motor center, and (2) concurrent activity in the positive pathway and the negative pathway leads to weakening of the connection between the positive pathway and the motor center.

measured by Western blotting (Griffith, unpublished observations). CaM kinase II may have nonenzymatic roles in neuronal function. It can act as a "calmodulin trap" after autophosphorylation (Meyer et al., 1992), and this may be important for frequency detection of calcium spikes (Hanson et al., 1994). Knockout of the $\alpha$-CaM kinase II gene in mice produces effects on plasticity that can be explained by a model invoking nonenzymatic properties of the kinase (Chapman et al., 1995). Decreasing the amount of kinase not only decreases activity, but it may be changing the distribution of kinase-binding proteins.

\section{A model for courtship conditioning}

Our results rule out certain classes of models for courtship conditioning (Fig. 6). A "serial" model in which the decrement of courtship of the mated female is required for memory formation is ruled out by the results of this study and that of Kane et al. (1997), showing memory without apparent learning. A "parallel" model in which the association forms and controls two separate output pathways for response to mated females and virgin females is also unlikely. Even when the male does not exhibit a decrement in his courtship of the trainer mated female, he can still respond normally to a mated female used as a tester.

Slightly more complicated circuits can be built that will repro- duce the essential features of the behavior. These models can be configured in a number of different ways, but all feature CaM kinase-dependent plasticity in a single circuit that has multiple output states. In the network model, courtship is turned on by signaling through a positive pathway responsive to both visual and stimulatory pheromonal cues. The decrement in courtship during exposure to a mated female is driven by the increase in strength of signal through an inhibitory pathway responsive to the aversive pheromone given off by mated females. The increase in strength of this pathway is attributable to a time- and courtshipdependent increase in production of the aversive pheromone by the mated female (Tompkins and Hall, 1981; Tompkins et al., 1983) and a CaM kinase-dependent heterosynaptic facilitation of the connection between this pathway and the courtship motor center (this synapse is indicated by gray shading in the negative pathway, Fig. 6). This pathway also modulates the positive pathway by causing a CaM kinase-dependent heterosynaptic inhibition of the connection between the positive pathway and the courtship motor center (this synapse is indicated by gray shading in the positive pathway, Fig. 6). Thus, over the course of the conditioning period, the wild-type male goes from a high level of courtship to a low level of courtship. Memory for conditioning is encoded in the weakening of the connection between the positive pathway and the motor center. When a virgin female is presented to a conditioned male, the positive pathway is too weak to turn on the courtship motor program.

Disruption of visual input has several effects on the circuit in wild-type flies. First, courtship stimulated by a virgin female is less robust because there is less positive input (Fig. 1). Second, the strength of the memory formed is decreased in the absence of visual input (compare memory in white and dim red light in Fig. $3 B, D)$. This result implies that the amount of activity in the stimulatory pathway can modulate the strength of heterosynaptic inhibition induced by the negative pathway.

Decreased CaM kinase activity produces impaired apparent learning in all cases tested. In this model, the decrease is produced by impairment of both the heterosynaptic inhibition and the facilitation of the circuit. The learning phenotype seen with inhibition of CaM kinase is not affected by visual input, suggesting that the negative pathway (which does not have a direct visual input) may be most important for apparent learning.

The effects of decreased CaM kinase activity on memory are more complicated and depend on the amount of activity in the positive pathway. If there is normal visual input, even in conditions in which CaM kinase activity is decreased, there is enough activity in the positive pathway to allow some memory formation (Fig. $3 B$ ). If vision is disrupted, the amount of activity in the positive pathway decreases, and consequently there is less heterosynaptic inhibition and no memory formation (Figs. 3B, Fig. $4 B, D)$.

The mechanisms invoked by this model have been elegantly demonstrated in vivo for the Aplysia gill- and siphon-withdrawal reflexes. Serotonergic input onto the presynaptic terminal of the sensory neuron causes facilitation of the connection between the sensory neuron and its motor neuron target, increasing neurotransmitter release (for review, see Byrne and Kandel, 1996). Application of FMRFamide to the presynaptic terminal causes inhibition of the sensory connection to the motor neuron and reduced transmitter release (Small et al., 1992). In Aplysia, both heterosynaptic facilitation and heterosynaptic inhibition have short- and long-term forms. The duration of the plasticity is determined by the strength of the stimulus and its temporal 
pattern. The network model for courtship conditioning also has this feature, and modification of the training paradigm for courtship conditioning can produce long-term memory (A. Uhimov and L. Tompkins, Temple University, personal communication; Joiner, unpublished observations).

In summary, we have shown that courtship conditioning in Drosophila has an important visual component. Manipulation of vision affects both basal courtship and memory formation. Visual input is important only during conditioning, indicating that its effect on memory is attributable to changes in the ability to form memory rather than defects in retrieval. Decreases in CaM kinase levels affect both the learning and memory phases of courtship conditioning. Memory defects are more apparent in visually defective flies, suggesting that $\mathrm{CaM}$ kinase is involved in memory formation and that visual inputs are integrated via a CaM kinasedependent process in courtship conditioning.

\section{REFERENCES}

Ackerman SL, Siegel RW (1986) Chemically reinforced conditioned courtship in Drosophila: responses to wild-type and the dunce, amnesiac and don giovanni mutants. J Neurogenet 3:111-123.

Brand AH, Perrimon N (1993) Targeted gene expression as a means of altering cell fates and generating dominant phenotypes. Development 118:401-415.

Byrne JH, Kandel ER (1996) Presynaptic facilitation revisited: state and time dependence. J Neurosci 16:425-435.

Chapman PF, Frenguelli BG, Smith A, Chen C-M, Silva AJ (1995) The $\alpha-\mathrm{Ca}^{2+} /$ calmodulin kinase II: a bidirectional modulator of plasticity. Neuron 14:591-597.

Chatterjee S, Singh BN (1988) Effect of light and dark on mating behavior of red eye and white eye Drosophila ananassae. Indian J Exp Biol 26:611-614.

Cho KO, Wall JB, Pugh PC, Ito M, Mueller SA, Kennedy MB (1991) The $\alpha$ subunit of type II $\mathrm{Ca}^{2+} /$ calmodulin-dependent protein kinase is highly conserved in Drosophila. Neuron 7:439-450.

Connolly K, Burnet B, Sewell D (1969) Selective mating and visual pigmentation: an analysis of the visual component in the courtship behavior of Drosophila melanogaster. Evolution 23:548-559.

Cook R (1980) The extent of visual control in the courtship tracking of Drosophila melanogaster. Biol Cybern 37:41-51.

DeJianne D, Pruzan-Hotchkiss A, Grossfeld J (1981) Light dependent mating inhibition in the white-eyed mutant of Drosophila pseudoobscura. Experientia 37:465-466.

Fischer JA, Giniger E, Maniatis T, Ptashne M (1988) GAL4 activates transcription in Drosophila. Nature 332:853-856.

Frank KD, Zimmerman WF (1969) Action spectra for phase shifts of a circadian rhythm in Drosophila. Science 163:688-689.

Gailey DA, Siegel RW (1989) A mutant strain in Drosophila melanogaster that is defective in courtship behavioral cues. Anim Behav 38:163-169.

Gailey DA, Lacaillade TC, Hall JC (1986) Chemosensory elements of courtship in normal and mutant, olfaction-deficient Drosophila melanogaster. Behav Genet 16:375-405.

Geer BW, Green MM (1962) Genotype, phenotype and mating behavior of Drosophila melanogaster. Amer Natur 96:175-181.

Griffith LC, Greenspan RJ (1993) The diversity of calcium/calmodulindependent protein kinase II isoforms in Drosophila is generated by alternative splicing of a single gene. J Neurochem 61:1534-1537.

Griffith LC, Verselis LM, Aitken KM, Kyriacou CP, Greenspan RJ (1993) Inhibition of calcium/calmodulin-dependent protein kinase in Drosophila disrupts behavioral plasticity. Neuron 10:501-509.

Griffith LC, Wang J, Zhong Y, Wu CF, Greenspan RJ (1994) Calcium/ calmodulin-dependent protein kinase II and potassium channel subunit Eag similarly affect plasticity in Drosophila. Proc Natl Acad Sci USA 91:10044-10048.

Grossfield J (1966) The influence of light on the mating behavior of Drosophila. Univ Tex Publ 6615:147-176.
Hanson PI, Meyer T, Stryer L, Schulman H (1994) Dual role of calmodulin in autophosphorylation of multifunctional CaM kinase may underlie decoding of calcium signals. Neuron 12:943-956.

Heisenberg M, Wonneberger R, Wolf R (1978) Optomotor-blind ${ }^{\mathrm{H} 31}$ : a Drosophila mutant of the lobula plate giant neurons. J Comp Physiol 124:287-296.

Hing ALY, Carlson JR (1996) Male-male courtship behavior induced by ectopic expression of the Drosophila white gene: role of sensory function and age. J Neurobiol 30:454-464.

Isono K (1993) Control of male courtship behavior by spectral light: involvement of retinular photoreceptors in the compound eyes of Drosophila. In: Sensory systems of arthropods (Wiese K, Gribakin FG, Popov AV, Renniger G, eds), pp 242-251. Basel: Birkhauser Verlag.

Jager RF, Fischbach K-F (1987) Some improvements on the Heisenberg-Bohl method for mass histology of Drosophila heads. Drosophila Information Service 66:162-165.

Kane NS, Robichon A, Dickinson JA, Greenspan RJ (1997) Learning without performance in PKC-deficient Drosophila. Neuron 18:307-314.

Lindsley DL, Zimm GG (1992) The genome of Drosophila melanogaster. San Diego: Academic.

Markow TA (1987) Behavioral and sensory basis of courtship success in Drosophila melanogaster. Proc Natl Acad Sci USA 84:6200-6204.

Martin J-R, Ollo R (1996) A new Drosophila $\mathrm{Ca}^{2+} /$ calmodulindependent protein kinase (Caki) is localized in the central nervous system and implicated in walking speed. EMBO J 15:1865-1876.

Meyer T, Hanson PI, Stryer L, Schulman H (1992) Calmodulin trapping by calcium-calmodulin-dependent protein kinase. Science 256: 1199-1202.

Pepling M, Mount S (1990) Sequence of a cDNA from the Drosophila melanogaster white gene. Nucleic Acids Res 18:1633.

Siegel RW, Hall JC (1979) Conditioned responses in courtship behavior of normal and mutant Drosophila. Proc Natl Acad Sci USA 76:565-578.

Small SA, Cohen TE, Kandel ER, Hawkins RD (1992) Identified FMRFamide-immunoreactive neuron LPL16 in the left pleural ganglion of Aplysia produces presynaptic inhibition of siphon sensory neurons. J Neurosci 12:1616-1627.

Sokal RR, Rohlf HR (1995) Biometry. New York: W. H. Freeman.

Spieth HT (1974) Courtship behavior in Drosophila. Annu Rev Entomol 19:383-406.

Stocker RF, Gendre N (1989) Courtship behavior of Drosophila genetically or surgically deprived of basiconic sensilla. Behav Genet 19:371-385.

Tompkins L (1984) Genetic analysis of sex appeal in Drosophila. Behav Genet 14:411-440.

Tompkins L, Hall JC (1981) The different effects on courtship of volatile compounds from mated and virgin Drosophila females. J Insect Physiol 27:17-21.

Tompkins L, Hall JC, Hall LM (1980) Courtship-stimulating volatile compounds from normal and mutant Drosophila. J Insect Physiol 26:689-697.

Tompkins L, Gross AC, Hall JC, Gailey DA, Siegel RW (1982) The role of female movement in the sexual behavior of Drosophila melanogaster. Behav Genet 12:295-307.

Tompkins L, Siegel RW, Gailey DA, Hall JC (1983) Conditioned courtship in Drosophila and its mediation by chemical cues. Genetics 13:565-578.

Villella A, Hall J (1996) Courtship anomalies caused by doublesex mutations in Drosophila melanogaster. Genetics 143:331-344.

Wang J, Renger J, Griffith LC, Greenspan RJ, Wu CF (1994) Concomitant alterations of physiological and developmental plasticity at $\mathrm{CaM}$ kinase II-inhibited synapses in Drosophila. Neuron 13:1373-1384.

Wehner R, Gartenmann G, Jungi T (1969) Contrast perception in eye color mutants of Drosophila melanogaster and Drosophila subobscura. J Insect Physiol 15:815-823.

Yin JC, DelVecchio M, Zhou H, Tully T (1995) CREB as a memory modulator: induced expression of a dCREB2 activator isoform enhances long-term memory in Drosophila. Cell 81:107-115. 